\title{
ON A FAMILY OF FOURIER TRANSFORMS
}

\section{AUREL WINTNER}

A procedure leading to specific properties of the symmetric stable distribution functions results by subjecting certain generalizations of the Bessel functions $J_{\alpha}(t)$ to a limit process. ${ }^{1}$ There arises the question as to the existence of the corresponding distributions in case the limit process involved is omitted. The object of this note is to delimit the conditions under which the answer is affirmative. It turns out that the situation is quite different from that resulting in the limiting case of stability.

Let $L(t ; \phi)$ denote the Fourier transform,

$$
L(t ; \phi)=\int_{-\infty}^{\infty} e^{i t x} d \phi(x), \quad-\infty<t<\infty,
$$

of a distribution function $\phi=\phi(x),-\infty<x<\infty$, that is, of a monotone function satisfying the boundary conditions $\phi(-\infty)=0$ and $\phi(\infty)=1$. If $\phi^{\prime}$ denotes the derivative of $\phi$ (a derivative which necessarily exists and is finite almost everywhere), the Stieltjes integral $L(t ; \phi)$ reduces to

$$
L(t ; \phi)=\int_{-\infty}^{\infty} e^{i t x} \phi^{\prime}(x) d x
$$

if and only if $\phi$ is absolutely continuous; in which case $\phi^{\prime}(x)$ is called the density of $\phi(x)$.

For a real or complex number $z$, let $z_{+}$denote $z$ or 0 according as $z$ is or is not positive. Thus, if $\lambda>0$ and $\mu>0$, the even function $L=\left(1-|t|^{\lambda}\right)^{\mu}{ }_{+}$of the real variable $t$ represents a continuous curve in a $(t, L)$-plane, this symmetric curve being situated above or on the $t$-axis according as $|t|<1$ or $1 \leqq|t|<\infty$. It should be noted for later reference that the curve has at $t=0$ a cusp with a tangent perpendicular to the $t$-axis, a corner with two distinct finite slopes or a tangent parallel to the $t$-axis, according as $\lambda<1, \lambda=1$ or $\lambda>1$, while $\mu$ is arbitrary.

If $\lambda=1=\mu$, the function $\left(1-|t|^{\lambda}\right)_{+}^{\mu}$ is $1-|t|$ or 0 according as $|t|<1$ or $|t| \geqq 1$, and represents therefore the Fourier transform $L(t ; \phi)$ of an absolutely continuous distribution function $\phi(x)$. This is

Received by the editors July 10, 1941

${ }^{1}$ A. Wintner, On a class of Fourier transforms, American Journal of Mathematics, vol. 58 (1936), pp. 45-90. 
seen either by subjecting the kernel of Dirichlet's factor of discontinuity to the process of auto-convolution or by applying a Fourier inversion. In fact,

$$
\int_{-\infty}^{\infty}\left(\frac{\sin x / 2}{x \pi^{1 / 2} / 2}\right)^{2} \cos t x \text { or } d x=\left\{\begin{array}{c}
1-|t| \text { if }|t| \leqq 1, \\
0 \text { if } 1 \leqq|t|<\infty
\end{array}\right.
$$

so that the density $\phi^{\prime}(x)$ is the square ()$^{2}$ on the left.

Thus, if $(\lambda, \mu)=(1,1)$, there exists a distribution function $\phi_{\lambda \mu}=\phi_{\lambda \mu}(x)$ such that

$$
L\left(t ; \phi_{\lambda \mu}\right)=\left\{\begin{array}{l}
\left(1-|t|^{\lambda}\right)^{\mu} \quad \text { if } \quad|t| \leqq 1, \\
0 \text { if } 1 \leqq|t|<\infty .
\end{array}\right.
$$

There arises the question for what additional pairs $(\lambda, \mu)$ does (1) determine a distribution function $\phi_{\lambda \mu}$. Since the derivative

$$
\phi_{11}^{\prime}(x)=(\sin x / 2)^{2} /\left(x \pi^{1 / 2} / 2\right)^{2},
$$

though non-negative, has zeros, no continuity consideration can indicate that there exists a distribution function $\phi_{\lambda \mu}$ for certain pairs $(\lambda, \mu)$ sufficiently close to $(1,1)$; in fact, the derivative $\phi_{\lambda \mu}^{\prime}(x)$ might then become negative, precluding a distribution function $\phi_{\lambda \mu}(x)$.

Since the function $\left(1-|t|^{\lambda}\right)^{\mu}+$ of $t$ is integrable over $(-\infty, \infty)$, it is clear by Fourier inversion that, if there exists at all a distribution function $\phi_{\lambda \mu}$ satisfying (1), then $\phi_{\lambda \mu}$ is absolutely continuous and has a density $\phi_{\lambda \mu}{ }^{\prime}(x) \geqq 0$ proportional to

$$
f_{\lambda \mu}(x)=\int_{0}^{1}\left(1-t^{\lambda}\right)^{\mu} \cos x t d t
$$

the factor of proportionality being $1 / \pi$ for arbitrary $(\lambda, \mu)$. Accordingly, the question can be restated by asking, which are those pairs $(\lambda, \mu)$ for which the function (3) satisfies the conditions represented by

$$
f_{\lambda \mu}(x) \geqq 0, \quad 0<x<\infty,
$$

and

$$
\int_{0}^{\infty} f_{\lambda \mu}(x) d x<\infty .
$$

If the unit of length on the $x$-axis is changed in the proportion $1: a$, the definition of the Fourier transform $L(t ; \phi)$ shows that the unit of length on the $t$-axis must be changed in the proportion $a: 1$. Hence, if $a=\mu^{1 / \lambda}$, the function (1) must be replaced by $\left(1-|t|^{\lambda} / \mu\right)^{\mu}$ if $|t| \leqq \mu^{1 / \lambda}$, 
and 0 if $|t| \geqq \mu^{1 / \lambda}$. This function of $t$ tends, as $\mu \rightarrow \infty$, to $\exp \left(-|t|^{\lambda}\right)$ for $-\infty<t<\infty$. This means that the limiting case $\phi_{\lambda \infty}$ of $\phi_{\lambda \mu}$ is the symmetric stable distribution function of index $\lambda$; a distribution function which is known to exist ${ }^{2}$ if and only if $\lambda$ does not exceed the critical value $\lambda=2$ belonging to a Gaussian distribution (the condition $f_{\lambda \infty}(x) \geqq 0$ corresponding to (4) being violated for certain values of $x$ if and only if $\lambda>2$ ).

It will turn out that the characteristic condition $\lambda \leqq 2$ belonging to $\mu=\infty$ must be replaced by $\lambda \leqq 1$ for every finite $\mu \geqq 1$. In other words, if $\mu$ is arbitrarily fixed on the range $1 \leqq \mu<\infty$, the distribution function $\phi_{\lambda \mu}$ does or does not exist according as $\lambda \leqq 1$ or $\lambda>1$. Incidentally, the trivial case (2) happens to be a limiting case, in the sense that (4), where $\lambda \leqq 1 \leqq \mu$, can be refined to

$$
f_{\lambda \mu}(x)>0, \quad 0<x<\infty
$$

unless $(\lambda, \mu)=(1,1)$.

It seems to be of interest that there exists a fixed lower bound (equal to 1) for the admissible values of $\mu$ in the whole admissible range of $\lambda(\leqq 1)$. That $\mu$ must be limited by some lower bound for every given admissible $\lambda$, is clear from Lévy's theory of indefinitely divisible laws. In fact, if $\lambda$ is arbitrarily fixed, (1) requires that $L\left(t ; \phi_{\lambda \mu}\right)$ be the $\mu$ th power of $L\left(t ; \phi_{\lambda 1}\right)$ for every $\mu$, although $L\left(t ; \phi_{\lambda 1}\right)$ vanishes for certain values of $t$. But this contradicts the stochastic theory just mentioned.

Actually, it is easy to see directly that there exists for every $\lambda>0$ a sufficiently small $\mu>0$ such that the corresponding function (3) becomes negative for certain values of $x$ and violates, therefore, (4). In fact, it is clear for reasons of continuity that there must exist for every $\lambda>0$ such a sufficiently small $\mu>0$, if it is true that, for every fixed $\lambda>0$, the function (3) becomes negative for certain values of $x$ in the limiting case $\mu=0$. But if $\mu=0$, then (3) reduces for every $\lambda>0$ to

$$
f_{\lambda 0}(x)=\int_{0}^{1} \cos x t d t=x^{-1} \sin x
$$

and attains therefore negative values.

For sake of simplicity, it will from now on be assumed that $\mu$ has a fixed value not less than 1 , while $\lambda>0$ is arbitrary.

It will be shown that (4) is violated or the necessary and sufficient condition, represented by (4) and (5) together, is satisfied, according

${ }^{2}$ For sharper results see, loc. cit., where references are given to the investigations of Lévy and Pólya. 
as $\lambda>1$ or $\lambda \leqq 1$; that is, according as the function on the right of (1) is or is not differentiable at $t=0$.

First, if $\lambda>2$, the function on the right of (1) is $1+o\left(t^{2}\right)$ as $t \rightarrow 0$. But this implies ${ }^{3}$ that either $(4)$ is violated or $L\left(t ; \phi_{\lambda \mu}\right)$ is independent of $t$, and the latter case is excluded by (1). Next, if $\lambda=2$, then (3) reduces to a constant multiple of the classical integral representation of the ratio $J_{\mu+1 / 2}(x) / x^{\mu+1 / 2}$; so that (4) is violated in view of the asymptotic formula of the Bessel functions for large real $x$. But a perfectly similar asymptotic formula, again with a sine function in the numerator, can be proved ${ }^{4}$ for (3) even if $\lambda<2$, provided that $\lambda>1$. Accordingly, (4) is violated ${ }^{5}$ whenever $\lambda>1$.

It will now be shown that (4) is satisfied whenever $\lambda \leqq 1$. To this end, it will be sufficient to prove (6), since the limiting case $(\lambda, \mu)$ $=(1,1)$ is taken care of by $(2)$.

Partial integration of (3) shows that

$$
x f_{\lambda \mu}(x)=\lambda \mu \int_{0}^{1}\left(1-t^{\lambda}\right)^{\mu-1} t^{\lambda-1} \sin x t d t .
$$

But, if $F_{\lambda \mu}(t)$ is an abbreviation for $\left(1-t^{\lambda}\right)_{+}^{\mu-1} t^{\lambda-1}$, the last integral can be written in the form

$$
\int_{0}^{\infty} F_{\lambda \mu}(t) \sin x t d t \equiv x^{-1} \sum_{n=0}^{\infty}(-1)^{n} \int_{n \pi / x}^{(n+1) \pi / x} F_{\lambda \mu}(t / x) \sin t d t
$$

for every $x>0$. Hence, in order to prove (6), it is sufficient to ascertain that the non-negative function $F_{\lambda \mu}(t / x)$ of $t / x$ is steadily decreasing as long as it does not vanish; in other words, that the function $\left(1-t^{\lambda}\right)^{\mu-1} t^{\lambda-1}$ of $t$ is steadily decreasing on the interval $0<t<1$. But both factors $\left(1-t^{\lambda}\right)^{\mu-1}, t^{\lambda-1}$ are positive and non-increasing, and at

${ }^{3}$ Loc. cit., p. 80.

${ }^{4} \mathrm{Cf}$. A. Wintner, On the asymptotic formulae of Riemann and of Laplace, Proceedings of the National Academy of Sciences, vol. 20 (1934), pp. 57-62.

${ }^{5}$ Naturally, the problem is less deep than to necessitate an actual application of the sharp asymptotic fact, referred to before. For instance, if $\mu=1$, a partial integration transforms (3) into

$$
f_{\lambda_{1}}(x)=\lambda x^{-1} \int_{0}^{1} t^{\lambda-1} \sin x t d t=\lambda x^{-1-\lambda} \int_{0}^{x} t^{\lambda-1} \sin t d t .
$$

But this function violates (4) whenever $\lambda>1$, since, if $\lambda-1>0$, the successive "waves"

$$
(-1)^{n} \int_{n \pi}^{(n+1) \pi} t^{\lambda-1} \sin t d t=\int_{n \pi}^{(n+1) \pi} t^{\lambda-1}|\sin t| d t, \quad \text { where } n=0,1,2, \cdots,
$$

increase with $n$. It is easy to transfer this primitive proof from $\mu=1$ to every $\mu>1$, if use is made of the second mean-value theorem. 
least one of them is decreasing, on the interval $0<t<1$. In fact, $\mu-1 \geqq 0$ and $\lambda-1 \leqq 0$ by assumption, where at least one of the two signs of equality is excluded by $(\lambda, \mu) \neq(1,1)$.

This proves (4) for $\lambda \leqq 1 \leqq \mu$. It remains to be shown that (5) is satisfied for $\lambda \leqq 1 \leqq \mu$. To this end, it is sufficient to verify that

$$
f_{\lambda \mu}(x)=O\left(x^{-1-\lambda}\right), \quad \text { as } x \rightarrow \infty .
$$

Suppose first that $\lambda=1$. Then (7) shows that (8) reduces to

$$
\int_{0}^{1}(1-t)^{\mu-1} \sin x t d t=O\left(x^{-1}\right), \quad \text { as } x \rightarrow \infty .
$$

But this estimate is clear from the second mean-value theorem.

In the remaining case, where $\lambda<1$, the estimate (8) can be refined to the asymptotic formula

$$
f_{\lambda \mu}(x) \sim \frac{\mu \Gamma(1+\lambda) \sin (\pi \lambda / 2)}{x^{1+\lambda}}, \text { as } x \rightarrow \infty ; \lambda<1 .
$$

In fact, since $0<\lambda<1$ implies the existence of the integral

$$
\int_{0}^{\infty} t^{\lambda-1} \sin t d t=\Gamma(\lambda) \sin (\pi \lambda / 2),
$$

it is clear from (7) that (9) is equivalent to the assertion that, as $x \rightarrow \infty$, the function

$$
x^{\lambda} \int_{0}^{1}\left(1-t^{\lambda}\right)^{\mu-1} t^{\lambda-1} \sin x t d t
$$

tends to the limit (10). This function can be written in the form

$$
\int_{0}^{x}\left(1-t^{\lambda} / x^{\lambda}\right)^{\mu-1} t^{\lambda-1} \sin t d t
$$

and tends therefore to the limit (10) if it is legitimate to carry out the process $x \rightarrow \infty$ beneath the last integral sign. But this is readily justified by the second mean-value theorem.

The JoIns Hopkins University 